\title{
Mechanisms of Alcoholic Pancreatitis
}

\section{Author/Contributor:}

Apte, Minoti; Pirola, Romano; Wilson, Jeremy

\section{Publication details:}

Journal of Gastroenterology and Hepatology

v. 25

Chapter No. 12

pp. 1816-1826

0815-9319 (ISSN)

\section{Publication Date:}

2010

\section{Publisher DOI:}

http://dx.doi.org/10.1111/j.1440-1746.2010.06445.x

\section{License:}

https://creativecommons.org/licenses/by-nc-nd/3.0/au/

Link to license to see what you are allowed to do with this resource.

Downloaded from http://hdl.handle.net/1959.4/53329 in https:// unsworks.unsw.edu.au on 2023-04-26 


\section{Mechanisms of Alcoholic Pancreatitis}

\section{Minoti V Apte, Romano C Pirola, Jeremy S Wilson}

Pancreatic Research Group, South Western Sydney Clinical School, Liverpool Hospital and

School of Medical Sciences, University of New South Wales, Sydney, Australia.

\section{Corresponding Author:}

Professor MV Apte

South Western Sydney Clinical School

Level 5, Wallace Wurth Building

The University of New South Wales

Sydney, NSW 2052

\section{AUSTRALIA}

Ph: $\quad 61-2-93858273$

Fax: 61-2-93851389

Email: m.apte@unsw.edu.au 


\begin{abstract}
Alcoholic pancreatitis is a major complication of alcohol abuse. The risk of developing pancreatitis increases with increasing doses of alcohol, suggesting that alcohol exerts dose-related toxic effects on the pancreas. However, it is also clear that only a minority of alcoholics develop the disease, indicating that an additional trigger may be required to initiate clinically evident pancreatic injury. It is now well established that alcohol is metabolised by the pancreas via both oxidative and non-oxidative metabolites. Alcohol and its metabolites produce changes in the acinar cells which may promote premature intracellular digestive enzyme activation thereby predisposing the gland to autodigestive injury. Pancreatic stellate cells (PSCs) are activated directly by alcohol and its metabolites and also by cytokines and growth factors released during alcoholinduced pancreatic necroinflammation. Activated PSCs are the key cells responsible for producing the fibrosis of alcoholic chronic pancreatitis. Efforts to identify clinically relevant factors that may explain the susceptibility of some alcoholics to pancreatitis have been underway for several years. An unequivocal, functionally characterised, association is yet to be identified in clinical studies, although in the experimental setting, endotoxin has been shown to trigger overt pancreatic injury and to promote disease progression in alcohol-fed animals. Thus, while the molecular effects of alcohol on the pancreas have been increasingly clarified in recent years, identification of predisposing or triggering factors remains a challenge.
\end{abstract}

Keywords : alcoholic pancreatitis, individual susceptibility, pancreatic fibrosis 


\section{Introduction}

Alcohol-related diseases represent a significant medical and social burden on society. One of the important digestive organs adversely affected by alcohol abuse is the pancreas. This was recognised as early as in 1878 by Freidreich ${ }^{1}$ who wrote "I am inclined to believe that a general chronic interstitial pancreatitis may result from excessive alcoholism (drunkard's pancreas)". While Freidreich's initial observation referred to the association of alcohol abuse with chronic pancreatic injury, it is now widely acknowledged that the clinical spectrum of alcohol-induced pancreatic injury ranges from isolated acute episodes to repeated acute attacks and finally to chronic manifestations.

The onset of alcoholic pancreatitis usually occurs in the 4th decade. The majority of patients are male and the average alcohol consumption in patients who develop pancreatitis is of the order of $150 \mathrm{~g}$ per day for a period of $10-15$ years prior to the initial presentation (alcoholic acute pancreatitis rarely occurs after a single binge). ${ }^{2}$ Typically, patients first present with acute abdominal pain, elevated serum levels of pancreatic enzymes and evidence of pancreatic injury in imaging studies. In severe cases, an acute presentation of alcoholic pancreatitis can be fatal. If the patient recovers but continues to drink, a chronic illness may ensue, characterised by i) persistent abdominal pain; and ii) manifestations of exocrine and endocrine insufficiency, namely maldigestion and diabetes, reflecting the irreversible histological changes of acinar atrophy and fibrosis (Figure 1).

In terms of the natural history of the disease, the traditional view amongst pancreatologists was that the condition was a form of chronic pancreatitis from the beginning, punctuated during its course by acute exacerbations. However, current opinion has reverted to the "necrosis-fibrosis" hypothesis, first proposed in 1946 by Comfort et $\mathrm{al}^{3}{ }^{3}$ that alcoholic pancreatitis begins as an acute process which progresses to chronic irreversible pancreatic 
damage as a consequence of repeated acute attacks (Figure 2). This idea is now well supported by several clinical and experimental studies. ${ }^{4-8}$

Over the past three decades there has been a concerted research effort to elucidate the mechanisms by which alcohol damages the pancreas. Major challenges in the field have been the relative inaccessibility of the human pancreas for analyses during life, and a lack of suitable animal models of the disease. Notwithstanding these difficulties, significant progress has been made in recent years with regard to our understanding of the cellular pathways mediating the acute toxic effects of alcohol on the exocrine pancreas and the progression of acute (potentially reversible) disease to chronic, irreversible changes in the gland.

\section{Pathogenesis of Alcoholic Pancreatitis}

Researchers investigating the pathogenesis of alcoholic pancreatitis have always been mindful of an apparent clinical paradox : on the one hand, the risk of developing pancreatitis is known to increase with increasing doses of alcohol, ${ }^{9-11}$ while on the other hand, epidemiological studies clearly show that only a minority of heavy drinkers develop clinically evident pancreatitis. $^{12,13}$ These observations indicate that although alcohol exerts constant, doserelated effects on the pancreas, additional 'triggers' or 'susceptibility factors' may play a role in initiating overt pancreatic disease. In view of this, two approaches have usually been used to study the mechanisms of alcohol-induced pancreatic damage, the first involving assessment of the direct effects of alcohol on the gland and the second related to identifying the susceptibility factors that may increase a heavy drinker's risk of developing clinical pancreatitis. 


\section{Direct toxic effects of alcohol on the pancreas}

\section{Effects of alcohol on pancreatic ducts}

Originally, most of the research on the effects of alcohol on the pancreas was focused on the sphincter of Oddi (SO), a direction that was inspired by Opie's early observations regarding the mechanism responsible for gallstone pancreatitis. ${ }^{14}$ These 'large duct/sphincteric' theories of pancreatitis included the biliary-pancreatic reflux theory, the duodeno-pancreatic reflux theory and the stimulation-obstruction theory. Each postulated that altered motility of the sphincter of Oddi (SO) in response to alcohol exposure played a central role in the development of alcoholic pancreatitis. However, studies in humans have yielded conflicting results; both decreased, ${ }^{15,16}$ and increased ${ }^{17,} 18$ sphincter of Oddi tone have been reported. Interestingly, recent animal studies appear to support a spasmogenic effect (increased SO tone) of alcohol on the sphincter. ${ }^{19}$ The resultant reduction in trans-sphincteric flow may partially explain the decrease in pancreatic secretion observed after acute alcohol intake in humans. $^{20}$

In the 1970 s, Sarles and colleagues ${ }^{11,21}$ put forward the intriguing theory that alcohol caused pancreatitis via precipitation of secreted proteins (protein plug formation) within small pancreatic ductules leading to acinar atrophy and fibrosis (Figure 2). In time, these plugs enlarged and calcified forming intraductal calculi. Thus, the focus of research shifted from the large ducts towards the small pancreatic ducts. It was postulated that contact of calculi with ductal epithelial cells led to ulceration, scarring, further obstruction and finally atrophy and fibrosis. Interestingly, in alcoholics there is an increased tendency to form precipitates in pancreatic juice. $^{22}$ The protein plug theory is further supported by experimental evidence indicating that alcohol intake alters two non-digestive enzyme components of pancreatic juice that may play a significant role in protein plug formation. These include (i) lithostathine (also called pancreatic stone protein), a 144 amino acid protein secreted by acinar cells which when 
hydrolysed by enzymes such as trypsin is converted to a highly precipitable 133 amino acid peptide called lithostathine S1. Messenger RNA levels of pancreatic lithostathine have been reported to be significantly increased in alcohol-fed rats; ${ }^{23}$ and ii) the glycoprotein GP2 which is the most abundant protein component of zymogen granule membranes. ${ }^{24}$ GP2 is secreted into pancreatic ducts via exocytosis from acinar cells along with digestive enzymes or released directly from apical plasma membranes via an enzymatic process. GP2 has unique properties for self-aggregation in pancreatic juice. Chronic alcohol administration to rats has been shown to significantly reduce pancreatic GP2 content ${ }^{25}$ possibly due to increased secretion of GP2 into the pancreatic juice, where it may form fibrillar aggregates that act as a nidus for protein and calcium precipitation.

Enhanced lithogenicity may also be secondary to increased viscosity of pancreatic secretions. In this regard, Sarles et $\mathrm{al}^{26}$ noted that patients with alcoholic pancreatitis display increased levels of sweat electrolytes suggestive of cystic fibrosis transmembrane regulator (CFTR) dysfunction. This could lead to viscous pancreatic secretions and significantly increase the risk of chronic changes in the gland. A role for ductular dysfunction in pancreatic injury is further supported by more recent reports of a strong association between mutations of the CFTR gene (which affect duct cell function), and the risk of developing idiopathic chronic pancreatitis. $^{27,} 28$ Thus, although an association between CFTR mutations and alcoholic pancreatitis has not been clearly demonstrated, it is reasonable to postulate that in addition to the acinar cell (see below), the duct cell is also an important site of alcohol-induced injury. Whether the ductal cell and protein plug formation play any role in the initiation of alcoholic pancreatitis is yet to be determined, but it is generally accepted that these events have the potential to facilitate disease progression. 


\section{Effects of alcohol on the acinar cell}

The acinar cell is the major functional unit of the exocrine pancreas. This cell is an enzyme factory which synthesises and secretes significant quantities of digestive enzymes in response to a meal. Researchers turned to the acinar cell for answers once it was evident that the sphincteric and small duct theories could not fully explain the pathogenesis of alcoholic pancreatitis. The concept that the acinar cell may be the site where alcohol-related pancreatic damage is initiated is not unreasonable given that digestive enzymes have a significant potential to cause tissue injury, particularly if activated prematurely within the acinar cell itself. The latter process is termed autodigestion.

\section{Role of digestive enzymes in pancreatic injury}

The digestive enzyme trypsinogen (a precursor form of trypsin) is usually activated in the duodenum by the enzyme enterokinase. However, in some circumstances, trypsinogen can also be activated by the lysosomal enzyme cathepsin B within acinar cells. ${ }^{29}$ In turn, active trypsin can activate other pro-enzymes and trigger a digestive enzyme activation cascade within the cell. Normally, the acinar cell is protected from digesting itself by synthesising most zymogens as inactive precursors, by segregating zymogens into membrane-bound organelles, and by intracellular anti-proteases. Disruption of these normal protective mechanisms can result in premature intracellular activation of digestive enzymes leading to autodigestive injury. Several lines of evidence support a role for digestive enzymes in pancreatic injury (see Apte et $\mathrm{al}^{30}$ for a review), including: i) development of pancreatic necrosis upon instillation of active digestive enzymes (including proteases, lipase and phospholipase) into the pancreatic duct; ii) presence of active digestive enzymes in ascitic fluid, pancreatic secretions and pancreatic tissue in both clinical and experimental pancreatitis; and iii) reduced severity of experimental pancreatitis upon administration of protease inhibitors. Studies in several models of experimental pancreatitis have now provided 
evidence to indicate that the initiating event in pancreatitis may be colocalisation of lysosomal enzymes and digestive enzymes in large vacuoles with subsequent activation of trypsinogen by the lysosomal enzyme cathepsin $\mathrm{B}$, or by autoactivation at the acidic $\mathrm{pH}$ in the vacuoles. The most convincing evidence in support of a role for digestive enzymes in pancreatic injury has come from the identification of mutations in the trypsinogen gene in patients with hereditary pancreatitis, ${ }^{28}$ a disease with a phenotype very similar to that of chronic alcoholic pancreatitis. Two trypsinogen mutations in particular, R122H and N29I, are gain-of-function mutations which lead to the synthesis of an altered form of trypsin that is resistant to inactivation. ${ }^{31,32}$

\section{Effect of alcohol on pancreatic enzymes}

Chronic alcohol administration to rats increases the pancreatic content of the digestive enzymes trypsinogen, chymotrypsinogen, and lipase, as well as the lysosomal enzyme cathepsin B. This increase in enzyme content is regulated at the mRNA level. ${ }^{33}$ Chronic alcohol intake has also been shown to reduce enzyme secretion by acinar cells secondary to acetaldehyde-induced microtubular dysfunction, ${ }^{34}$ and/or alcohol-induced reorganisation of the apical cytoskeleton in acinar cells; ${ }^{35}$ both these effects can perturb exocytosis and cause cellular accumulation of enzymes.

In addition, alcohol administration decreases the stability of the membranes of zymogen granules and lysosomes, the organelles that contain digestive and lysosomal enzymes, respectively. The observed effect of alcohol on lysosomal stability may be mediated by cholesteryl esters ${ }^{36}$ and fatty acid ethyl esters, ${ }^{37}$ substances known to accumulate in the pancreas after chronic alcohol consumption,. ${ }^{38,39}$ The alcohol-induced instability of zymogen granules is postulated to be the result of loss of a glycoprotein GP2, which is important to the shape and stability of the granules. ${ }^{25}$ 
Thus, chronic alcohol exposure results in an acinar cell that has significantly increased levels of digestive and lysosomal enzymes accompanied by decreased stability of the organelles that contain these enzymes. These changes increase the potential for contact between digestive and lysosomal enzymes. In the presence of an appropriate trigger factor, premature intracellular activation of digestive enzymes can occur, leading to autodigestive injury of the gland.

\section{Metabolism of alcohol by pancreatic acinar cells}

The capacity of the pancreas to metabolise alcohol has now been well documented. ${ }^{40-42}$ The pancreas can metabolise alcohol via both oxidative and non-oxidative pathways yielding the toxic metabolites acetaldehyde and fatty acid ethyl esters, respectively. The oxidative pathway is catalysed by the enzyme alcohol dehydrogenase (ADH) with contributions from cytochrome P4502E1 (CYP2E1) and, to a lesser extent, from catalase. Using rat pancreatic acinar cells, ADH activity in the pancreas was shown to be resistant to inhibition by 4methylpyrazole (4-MP, a specific inhibitor of ADH I) and consistent with the kinetics of ADH III (a non-saturable isoform of ADH with low affinity and a high $\mathrm{Km}$ for alcohol). ${ }^{41}$ However, a recent study using human pancreatic tissue has reported that the predominant class of $\mathrm{ADH}$ in human pancreatic acini is $\mathrm{ADH}$, with $\mathrm{ADH}$ III contributing little to pancreatic alcohol oxidation. ${ }^{43}$ These disparate findings may reflect species differences in terms of kinetic properties for ADH isozymes. CYP2E1 has also been identified in the pancreas, and, as in the liver, has been shown to be inducible by chronic alcohol feeding. ${ }^{44}$ However the role of CYP2E1 in pancreatic alcohol metabolism remains to be fully clarified.

Oxidative alcohol metabolism results in the generation of reactive oxygen species (ROS) as a by-product ${ }^{45}$, and at the same time, depletion of the ROS scavenger glutathione. ${ }^{46}$ The resulting imbalance between the production of ROS (which can damage lipid membranes, 
intracellular proteins and DNA) and the proteins responsible for their removal (glutathione, glutathione peroxidase, superoxide dismutase, catalase) leads to oxidant stress. Evidence of increased oxidant stress has been reported in both humans with alcoholic pancreatitis and experimental animals receiving alcohol diets. ${ }^{47-49}$

The non-oxidative pathway of alcohol metabolism involves the esterification of alcohol with fatty acids to form fatty acid ethyl esters, a reaction catalysed by fatty acid ethyl ester synthases (FAEE synthases). These enzymes have not yet been fully characterised or cloned, but possible candidates include carboxylester lipase and triglyceride lipase. It is interesting to note that the pancreas exhibits FAEE synthase activity that is several fold higher than that observed in the liver, ${ }^{40}$ and that FAEEs have been shown to accumulate in human and rat pancreas after alcohol intake. ${ }^{38,40,42,50}$ Importantly, the concentrations of FAEEs generated in the pancreas of alcohol-fed rats are similar to those known to induce damage in vitro to subcellular organelles of pancreatic acinar cells. ${ }^{37,42}$

Given that both pathways of alcohol metabolism are evident in the pancreas, the possibility of a linkage between the two pathways has been examined by researchers, using both in vitro and in vivo approaches. Werner and his colleagues ${ }^{50,51}$ have reported that the formation of FAEEs in isolated pancreatic acini was increased in the presence of inhibitors of oxidative metabolism, compared to that in the absence of the inhibitors. Similarly, they found that in vivo infusion of alcohol with inhibitors of oxidative metabolism led to increased FAEE accumulation in rat pancreas. However, whether alcohol oxidation in the pancreas was indeed significantly inhibited by the infused inhibitors was not assessed in these studies.

In terms of the relative contribution of the oxidative and non-oxidative pathways to alcohol metabolism in the pancreas, the predominant pathway is reported to be the former. ${ }^{40,42}$ Thus, 
for the same concentration of alcohol, tissue levels of acetate (a product of alcohol oxidation) were found to be significantly higher than those of FAEEs (products of non-oxidative alcohol metabolism). However, this does not diminish the potential importance of the non-oxidative pathway of alcohol metabolism in the pancreas because, as noted earlier, tissue levels of the products (FAEEs) generated by this pathway have been shown to be sufficient to cause pancreatic injury.

\section{Pancreatic injury caused by toxic metabolites of alcohol}

The products of alcohol oxidation (acetaldehyde and ROS) and those of non-oxidative metabolism of alcohol (FAEEs) have all been reported to cause acinar cell injury. In vivo studies have shown that acetaldehyde (albeit at high concentrations) causes morphological damage to rat and dog pancreas. ${ }^{52}$ As noted earlier, acetaldehyde has also been reported to inhibit CCK-stimulated acinar cell secretion, most likely via microtubular dysfunction and disruption of the actin cytoskeleton (effects which inhibit exocytosis) and via disruption of the binding of secretagogues to their receptor. ${ }^{53}$ Another metabolic consequence of alcohol oxidation that could contribute to acinar injury is an alteration in the intracellular redox state of the acinar cell (a reduced $[\mathrm{NAD}] /[\mathrm{NADH}]$ ratio and increased [lactate]/[pyruvate] ratio) secondary to increased production of hydrogen ions (reducing equivalents). ${ }^{54}$

Both clinical and experimental studies have demonstrated that alcohol intake causes oxidant stress within the pancreas, ${ }^{47-49}$ which is thought to play a role in the observed alcohol-induced destabilisation of zymogen granules and lysosomes. Antioxidant therapy has been shown to

ameliorate clinical as well as experimental pancreatitis, ${ }^{55}$ further supporting the role of oxidant stress in pancreatic injury. 
Several studies have now demonstrated that the non-oxidative metabolites of alcohol, FAEEs have the capacity to damage the pancreas. Infusion of FAEEs in rats causes pancreatic oedema, acinar cell vacuolisation, trypsinogen activation and increased extracellular matrix protein levels in the gland. ${ }^{56,57}$ The latter effect may be of relevance to the development of alcohol-induced pancreatic fibrosis (vide infra). FAEEs, at concentrations known to occur in vivo, destabilise lysosomes in pancreatic acinar cells. ${ }^{37}$ This effect may increase the potential for contact between lysosomal enzymes and digestive enzymes, facilitating premature intracellular activation of the latter.

The mechanisms by which FAEEs exert their toxic effects have received considerable attention in recent years. Candidate pathways include : i) direct interaction of the compounds with cellular membranes; ${ }^{58}$ ii) stimulation of cholesteryl ester synthesis by transesterification; ${ }^{59}$ and iii) release of free fatty acids by hydrolysis of FAEEs, a process thought to contribute to FAEE-induced mitochondrial damage. ${ }^{59}$

There has also been a growing interest in recent years in the signalling pathways that may mediate the observed effects of alcohol and its metabolites on acinar cells. In this regard, the transcription factors NF- $\mathrm{KB}$ and AP-1 (important regulators of cytokine expression) have been shown to be induced by alcohol and acetaldehyde as well as by FAEEs. ${ }^{40}$ Perturbations of intracellular calcium, an important second messenger, have also been reported in response to alcohol and FAEEs. Criddle and colleagues ${ }^{60}$ have shown that exposure of pancreatic acinar cells in vitro to the FAEE palmitoleic acid ethyl ester (PAEE) cause a sustained rise in cytosolic calcium secondary to increased calcium release from intracellular sources such as the endoplasmic reticulum (via stimulation of inositol triphosphate receptors) and decreased clearance of calcium due to dysfunction of the calcium ATPase pumps in the endoplasmic reticulum and plasma membrane. The ATPase pump 
dysfunction is dependent on the hydrolysis of PAEE to its free fatty acid palmitoleic acid, which leads to uncoupled mitochondrial oxidative phosphorylation and deficient ATP production.

A recent study by Castillo-Vaquero et $\mathrm{al}^{61}$ suggests an additional source for raised intracellular calcium in alcohol-exposed acinar cells. These authors have shown a significant increase in the influx of extracellular calcium into mouse acinar cells incubated with physiological concentrations of the secretagogue cholecystokinin $(\mathrm{CCK})+$ intoxicating concentrations of alcohol $(50 \mathrm{mM})$. Inhibition of alcohol oxidation by the ADH inhibitor 4 methylpyrazole, or preincubation with the antioxidant cinnamtannin-B prevented the alcoholinduced calcium influx. These findings indicate that alcohol oxidation and the subsequent generation of ROS may play an important role in this process. It is thought that the sustained rise in intracellular calcium leads to mitochondrial calcium overload and mitochondrial depolarisation, eventually causing acinar cell death.

\section{Alcohol-induced pancreatic fibrosis}

As noted earlier, alcoholic pancreatitis exhibits both acute and chronic manifestations. Pancreatic fibrosis represents a major histological characteristic of alcohol-induced chronic pancreatic injury. Over the past two decades significant advances have been made in our understanding of the mechanisms responsible for pancreatic fibrosis, and the central role of pancreatic stellate cells (PSCs) in the fibrogenic process is now firmly established. ${ }^{62}$

PSCs are located at the basolateral aspect of acinar cells. In health, they display a quiescent phenotype characterised by the presence of vitamin-A containing lipid droplets in the cytoplasm (Figure 3), and positive immunostaining for cytoskeletal proteins such as desmin

(Figure 4) and glial acidic fibrillary protein ${ }^{63,64}$. In the normal pancreas, PSCs regulate 
extracellular matrix turnover via their ability to synthesise matrix proteins as well as matrix degrading enzymes (matrix metalloproteinases, MMPs). During pancreatic necroinflammation, PSCs become activated and assume a myofibroblast-like phenotype characterised by the loss of vitamin A droplets, production of $\alpha$-smooth muscle actin $(\alpha-$ SMA), and increased synthesis of extracellular matrix proteins such as collagens I and III, fibronectin and laminin. The resulting imbalance between matrix synthesis and matrix degradation ultimately leads to the development of pancreatic fibrosis. Factors known to be upregulated during pancreatic injury such as TGF- $\beta$, platelet-derived growth factor (PDGF) and proinflammatory cytokines stimulate PSC proliferation and production of extracellular matrix proteins. ${ }^{62,65-67}$ Activated PSCs can also secrete endogenous cytokines resulting in an autocrine loop of cell activation. Notably, activated PSCs also produce increased amounts of MMP2, ${ }^{68}$ which is known to degrade collagen IV, an essential component of the basement membrane. This facilitates the deposition of fibrillar collagen observed in pancreatic fibrosis.

In terms of alcohol-induced chronic pancreatitis, it is relevant to emphasise that both rat and human PSCs have been shown to be activated by exposure to clinically relevant concentrations of alcohol ranging from $10 \mathrm{mM}$ (encountered during social drinking) to 50 $\mathrm{mM}$ (seen with heavy alcohol consumption). ${ }^{69,70}$ Rat and human PSCs are also activated by acetaldehyde at concentrations of 150 and $200 \mu \mathrm{M}$; these levels are higher than mean blood levels reported in alcoholics, but similar to liver tissue concentrations reported in alcohol-fed rats. ${ }^{69,70}$ Alcohol and acetaldehyde increase the secretion of MMP2 by PSCs. ${ }^{68}$ Interestingly, alcohol has recently been reported to stimulate the synthesis of endogenous cytokines such as interleukin 8 and connective tissue growth factor, respectively by human ${ }^{70}$ and mouse ${ }^{71}$ PSCs.Such endogenous cytokines could activate their own receptors on PSC membranes via autocrine pathways to further perpetuate PSC activation. Most recently, alcohol has been 
shown to inhibit PSC apoptosis, thereby facilitating prolonged survival of activated cells in the $_{\text {pancreas. }^{72}}$

There is evidence to suggest that PSCs have the capacity to metabolise alcohol via the oxidative pathway. Rat PSCs exhibit ADH activity, which is induced by exposure to $50 \mathrm{mM}$ alcohol. ${ }^{69}$ Exposure to 4-MP abolishes ADH activity in the cells, indicating that ADH1 is the isoform in PSCs. Furthermore, incubation of the cells with alcohol in the presence of 4-MP prevents their activation, suggesting that PSC activation is driven by the conversion of alcohol to acetaldehyde rather than by alcohol itself. Furthermore, alcohol metabolism causes oxidant stress in the cells, while alcohol-induced PSC activation can be prevented by the antioxidant vitamin E. These observations in rat PSCs are well supported by a recent study reporting activity of an ADH I isozyme, namely ADH1C in quiescent human PSCs which was inhibited by the ADH1 inhibitor 4-MP. ${ }^{43}$ Interestingly, the study showed that the expression of ADH1C was increased in activated human PSCs in chronic pancreatitis and pancreatic cancer sections. $^{43}$

Another possible activator for PSCs that is relevant to alcoholic pancreatitis is bacterial endotoxin. As detailed below, a recent study has demonstrated a key role for lipopolysaccharide (LPS), an endotoxin found in the cell wall of Gram negative bacteria such as E coli, in the initiation and progression of alcoholic pancreatitis. ${ }^{73}$ Similar to alcohol, LPS has been shown to activate PSCs (as assessed by $\alpha$ SMA expression) and to inhibit PSC apoptosis. Importantly, alcohol and LPS together exert synergistic effects on PSC activation and $_{\text {apoptosis. }}{ }^{72}$

In summary, alcohol exposure activates PSCs via both direct effects of alcohol and its metabolites, as well as by indirect pathways (effects of proinflammatory cytokines and LPS). 
PSC activation leads to the synthesis of excessive amounts of ECM proteins and the development of pancreatic fibrosis.

\section{Individual Susceptibility to Alcoholic Pancreatitis}

Despite the abundance of experimental evidence supporting direct toxic effects of alcohol and its metabolites on the pancreas, which is likely the case in all heavy drinkers, it is well established that only a minority of alcoholics develop pancreatitis. This fact clearly indicates that an additional insult (second hit) or individual susceptibility factor is required for a clinical attack of pancreatitis to be triggered in heavy drinkers. The search for this trigger factor/cofactor/susceptibility factor has prompted numerous studies, as summarized in Table 1.

Ideally, studies into individual susceptibility to alcoholic pancreatitis should compare alcoholics with the disease and alcoholics without the disease so that the index and the control groups differ in only one variable, i.e. the presence or absence of pancreatitis. This has not always been the case, with several studies using only the healthy population as a control group.

Numerous susceptibility factors have been examined to date including diet, amount and type of alcohol consumed, the pattern of drinking, lipid intolerance and smoking (see reviews ${ }^{74}$, ${ }^{75}$ ). The role of smoking as a trigger factor for alcoholic pancreatitis has been a particularly fraught subject (see reviews ${ }^{74,76}$ ). This is partly because a significant proportion of heavy drinkers are also smokers, making it difficult to demonstrate unequivocally an independent role of smoking in the initiation of pancreatitis. After adjusting for alcohol and other risk factors, a recently published study concluded that smoking is independently associated with

chronic pancreatitis. ${ }^{77}$ However, as acknowledged by the authors themselves, the retrospective nature of the study made it difficult to accurately stratify the extent of smoking 
and alcohol use. Furthermore, the study cohort included patients with chronic pancreatitis of a variety of aetiologies; only a small proportion of these could be classified as heavy drinkers. While the role of smoking as an initiating factor in alcoholic pancreatitis remains uncertain, there is some evidence to suggest that it may facilitate the progression of the disease by promoting the development of pancreatic calcifications and endocrine dysfunction. ${ }^{78}$

Another recently explored risk factor for alcoholic pancreatitis is obesity. ${ }^{79}$ Using a prospectively recruited cohort of 227 patients with alcoholic chronic pancreatitis and age- and sex-matched healthy subjects as controls, Ammann et al ${ }^{79}$ reported that obesity (prior to onset of chronic pancreatitis, defined as body mass index $[\mathrm{BMI}]>30$ ) was 5 -fold more frequent in patients with alcoholic chronic pancreatitis compared to healthy controls, but had no effect on disease progression. However, an earlier study has reported that obesity is highly prevalent in asymptomatic alcoholics compared to the general population. ${ }^{80}$ This observation and the lack of an appropriate control group (alcoholics without pancreatitis) in the study by Ammann and colleagues ${ }^{79}$ preclude any definitive conclusions regarding obesity as a susceptibility factor for the development of alcoholic pancreatitis. Thus, in terms of 'environmental' factors, a clear susceptibility factor for alcoholic pancreatitis remains to be identified.

A number of hereditary factors have also been examined as possible triggers for alcoholic pancreatitis. These include blood group antigens, HLA serotypes, alpha-1-antitrypsin phenotypes, CFTR genotypes, the cytokines transforming growth factor beta (TGF $\beta$ ), tumour necrosis factor $\alpha(\mathrm{TNF} \alpha)$, interleukin 10 and interferon gamma, genotypes of alcohol metabolising enzymes [alcohol dehydrogenase (ADH), aldehyde dehydrogenase (ALDH) and cytochrome P4502E1 (CYP2E1)] and detoxifying enzymes such as UDP glucuronosyltransferase (UGT1A7) and glutathione S-transferase. Most studies have failed to show any association of these genes with alcoholic pancreatitis, although one study reported a 
positive association between the risk of developing alcoholic pancreatitis and a polymorphism of the gene for one of the candidate FAEE synthase enzymes, carboxylester lipase (CEL). ${ }^{81}$ The functional significance of this polymorphism has not yet been elucidated.

Mutations of genes related to digestive enzymes and their inhibitors, including trypsinogen, chymotrypsinogen, secretory trypsin inhibitor (PSTI) also known as serine protease inhibitor Kazal type 1 (SPINK-1), mesotrypsin and enzyme Y, have also been examined. Recently, a protective variant (G191R) of the anionic trypsinogen gene PRSS2, which results in a form of trypsin that is easily degraded, was reported to be significantly less common in patients with alcoholic chronic pancreatitis compared to healthy controls, but the prevalence of this variant in alcoholics without pancreatitis was not tested. ${ }^{82}$ An association between mutated SPINK1 and alcoholic pancreatitis has also been described. The N34S mutation, a c.101A $>\mathrm{G}$ transition leading to substitution of asparagine by serine at codon 34 , was found in $5.8 \%$ patients with alcoholic pancreatitis, compared to $1.0 \%$ alcoholic controls without pancreatitis. ${ }^{83}$ A more recent study on Romanian patients has reported that $5 \%$ of patients with ACP had the N34S mutation compared to $1 \%$ of healthy controls. ${ }^{84}$ However, since the N34S mutated human SPINK1 does not show any altered trypsin inhibitor capacity, ${ }^{85}$ the functional consequences of this mutation are unclear. Therefore, the pathogenic significance of mutated SPINK1 in alcoholic pancreatitis remains unknown.

In experimental settings, other trigger factors for alcoholic pancreatitis have been investigated. Administration of supraphysiological doses of cholecystokinin (CCK) to rats receiving alcohol via gastrostomy catheters has induced pancreatic injury. ${ }^{86}$ Perides et $\mathrm{al}^{7}$ confirmed this effect in alcohol-fed mice and have also shown that the pancreatic injury progresses to fibrosis after repeated injections of the synthetic CCK analogue, caerulein. Although these findings are interesting, the clinical relevance of $\mathrm{CCK}$ as a trigger factor is 
questionable, since the amounts of CCK used in these studies were orders of magnitude higher than the levels observed in vivo in humans.

A potential co-factor that does have relevance to the clinical situation is bacterial endotoxaemia. It is well established that serum endotoxin levels are increased in alcoholics, even after a single binge, most likely due to the alcohol-induced increase in gut permeability permitting translocation of gram-negative bacteria (such as E. coli) across the mucosal barrier and decreased clearance of endotoxin by Kupffer cells in the liver. ${ }^{87,} 88$ Interestingly, endotoxaemia is predictive of the severity of acute pancreatitis, regardless of aetiology, ${ }^{89}$ but its role in alcoholic pancreatitis per se has only recently attracted attention. A study by Vonlaufen et $\mathrm{al}^{73}$ provided convincing evidence that endotoxin (LPS) challenge in alcohol-fed rats initiates overt pancreatic injury; it also stimulates progression to chronic disease manifesting as acinar atrophy and fibrosis (Figure 5).

To summarise, although clinical studies have yet to convincingly identify a susceptibility factor for alcoholic pancreatitis, recent experimental findings have indicated that bacterial endotoxin is a promising candidate. Thus, genetic polymorphisms of the proteins that regulate the effects of endotoxin in the body such as the LPS receptor TLR4, and the adapter proteins CD14 and MD2 are worthy of study. Other possible susceptibility factors that have not yet been examined fully include proteins relevant to cellular anti-oxidant defences, and minor CFTR mutations.

\section{Is alcoholic pancreatitis reversible?}

A major unanswered question in the pathophysiology of alcoholic pancreatitis is whether the condition is reversible. Clinicians routinely advise patients to abstain from alcohol, but there is a paucity of evidence from epidemiological studies about the benefit of abstinence on 
alcoholic pancreatitis. Lankisch and colleagues ${ }^{2}$ have reported that alcohol abstinence does not significantly benefit pain in patients with alcoholic pancreatitis. Gullo et al ${ }^{90}$ noted that although pancreatic function continued to deteriorate in patients that continued to drink as well as in abstainers, the rate of deterioration was slower in the abstinent group.

A recent experimental study may help shed some light on this issue. Using the model of alcoholic pancreatitis produced by LPS challenge in alcohol-fed rats, Vonlaufen et al ${ }^{72}$ have assessed the impact of withdrawal or continuation of alcohol on established pancreatitis. They have shown that, in contrast to the persistence of established injury upon continuation of alcohol, withdrawal of alcohol for as short a period as 3 days resulted in a significant reduction in the indices of pancreatic injury (edema, hemorrhage, acinar cell vacuolisation and acinar necrosis). There was complete reversal of damage at 7 days. Importantly, pancreatic fibrosis also decreased with alcohol withdrawal, with complete resolution at 3 weeks of abstinence (Figure 6). The beneficial effect on fibrosis is likely related to loss of alcohol-induced inhibition of PSC apoptosis noted earlier. This enables the deletion of activated PSCs through cell death, and halts the fibrogenic process. It must be acknowledged here that the above studies describe reversal of early alcohol-induced pancreatic injury. Nonetheless, the findings are important because they provide a critical platform for future studies on reversibility of more extensive disease.

\section{$\underline{\text { Conclusion }}$}

From the available literature discussed above, the current concepts about the pathogenesis of alcoholic pancreatitis can be summarised (Figure 7) as follows: 
Alcohol is metabolised by pancreatic acinar and stellate cells to yield oxidative and nonoxidative metabolites and metabolic by-products (ROS). In acinar cells, alcohol and/or its metabolites exert effects which lead to:

i) increased digestive and lysosomal enzyme content within acinar cells (via increased synthesis, and possibly decreased secretion in the case of digestive enzymes); and

ii) increased potential for contact between digestive and lysosomal enzymes secondary to increased organelle fragility mediated by compounds such as cholesteryl esters, FAEEs and ROS.

These changes increase the likelihood of premature intracellular activation of digestive enzymes. In the presence of a trigger factor, such as bacterial endotoxin, the gland is predisposed to autodigestive injury and necroinflammation.

Parallel processes that may act in a synergistic manner with the above changes include : i) increased cytokine production secondary to activation of transcription factors $(\mathrm{NF}-\kappa \mathrm{B}$ and AP1) in acinar cells, and ii) sustained increases in cytosolic calcium levels leading to mitochondrial calcium overload, mitochondrial depolarisation and cell death.

In pancreatic stellate cells, alcohol, its metabolites and metabolic by-products lead to:

i) cell activation and abnormal synthesis of ECM proteins;

ii) decreased apoptosis leading to prolonged survival of activated PSCs; and

iii) synthesis of endogenous cytokines and growth factors, possibly secondary to activation of the transcription factor, activator protein-1 (AP-1). In addition, PSCs are activated in a paracrine manner by cytokines released by acinar cells and infiltrating inflammatory cells during alcohol-induced necroinflammation. Activated PSCs then drive the development of pancreatic fibrosis. 
Once initiated, progression of pancreatic injury to chronic damage may be determined by continued alcohol intake, environmental factors such as smoking and the frequency of repeated episodes of necroinflammation. On the other hand, regression/reversal of established alcoholic pancreatitis may be possible, at least in the early stages, after discontinuation of alcohol. In this respect, the study reported by Vonlaufen and colleagues ${ }^{72}$ provides the first experimental evidence to support the intuitive clinical advice given to patients with alcoholic pancreatitis to abstain from alcohol.

Acknowledgements : The authors are supported by project grant funding from the National Health and Medical Research Council of Australia. 


\section{References}

[1] Freidreich N. Disease of the pancreas. New York: William Wood 1878.

[2] Lankisch PG, Lohr-Happe A, Otto Jet al. Natural course in chronic pancreatitis. Pain, exocrine and endocrine pancreatic insufficiency and prognosis of the disease. Digestion 1993; 54: 148-55.

[3] Comfort HW, Gambill EE, Baggenstoss AH. Chronic relapsing pancreatitis: a study of 29 cases without associated disease of the biliary or gastrointestinal tract. Gastroenterology 1946; 6: 239-85.

[4] Ammann RW, Muellhaupt B. Progression of alcoholic acute to chronic pancreatitis. Gut 1994; 35: 552-6.

[5] Deng X, Wang L, Elm MSet al. Chronic alcohol consumption accelerates fibrosis in response to cerulein-induced pancreatitis in rats. Am J Pathol 2005; 166: 93-106.

[6] Elsasser HP, Haake T, Grimmig Met al. Repetitive cerulein-induced pancreatitis and pancreatic fibrosis in the rat. Pancreas 1992; 7: 385-90.

[7] Perides G, Tao X, West Net al. A mouse model of ethanol dependent pancreatic fibrosis. Gut 2005; 54: 1461-7.

[8] Renner IG, Savage WT, Pantoja JLet al. Death due to acute pancreatitis. Digestive Diseases and Sciences 1985; 30: 1005-18.

[9] Bourliere M, Barthet M, Berthezene Pet al. Is tobacco a risk factor for chronic pancreatitis and alcoholic cirrhosis? Gut 1991; 32: 1392-5.

[10] Durbec JP, Sarles H. Multicenter survey of the etiology of pancreatic diseases. Relationship between the relative risk of developing chronic pancreatitis and alcohol, protein and lipid composition. Digestion 1978; 18: 337-50.

[11] Sarles H. Alcoholism and pancreatitis. Scandinavian Journal of Gastroenterology 1971; 6: 193-98.

[12] Dreiling DA, Koller M. The natural history of alcoholic pancreatitis: update 1985. Mount Sinai Journal of Medicine 1985; 52: 340-2.

[13] Steinberg W, Tenner S. Acute pancreatitis. New England Journal of Medicine 1994; 330: 1198-210.

[14] Opie EL. The etiology of acute haemorrhagic pancreatitis. Bulletin of John Hopkins Hospital 1901; 12: 182-8.

[15] Goff JS. The effect of alcohol in the pancreatic duct sphincter of Oddi. American Journal of Gastroenterology 1993; 88: 656-60.

[16] Viceconte G. Effect of ethanol on sphincter of Oddi: an endoscopic manometric study. Gut 1983; 24: 20-7.

[17] Pirola RC, Davis AE. Effects of intravenous alcohol on motility of the duodenum and the sphincter of Oddi. Australian Annals of Medicine 1970; 19: 24-9.

[18] Yamasaki K, Okazaki K, Sakamoto Yet al. Effects of ethanol on the motility of papillary sphincter and exocrine pancreas in the monkey. American Journal of Gastroenterology 1993; 88: 2078-83.

[19] Sonoda Y, Woods CM, Toouli Jet al. Intragastric ethanol reduces sphincter of Oddi function in the anaesthetised Australian possum. Pancreas 2005; 31: 469.

[20] Hajnal F, Flores MC, Radley Set al. Effect of alcohol and alcoholic beverages on meal-stimulated pancreatic secretion in humans. Gastroenterology 1990; 98: 191-6.

[21] Sarles H. Chronic calcifying pancreatitis - chronic alcoholic pancreatitis. Gastroenterology 1974; 66: 604-16.

[22] Guy O, Robles DG, Adrich Zet al. Protein content of precipitates present in pancreatic juice of alcoholic subjects and patients with chronic calcifying pancreatitis. Gastroenterology 1983; 84: 102-7. 
[23] Apte MV, Norton ID, Haber PSet al. Both ethanol and protein deficiency increase messenger RNA levels for pancreatic lithostathine. Life Sciences 1996; 58: 485-92.

[24] Rindler MJ, Hoops TC. The pancreatic membrane protein GP2 localises specifically to secretory granules and is shed into the pancreatic juice as a protein aggregate. European Journal of Cell Biology 1990; 53: 154-63.

[25] Apte MV, Norton ID, Haber PSet al. Chronic ethanol administration decreases rat pancreatic GP2 content. Biochemica et Biophysica Acta 1997; 1336: 89-98.

[26] Sarles H, Sarles JC, Camatte Ret al. Observations on 205 confirmed cases of acute pancreatitis, recurring pancreatitis, and chronic pancreatitis. Gut 1965; 6: 545-59.

[27] Cohn JA, Neoptolemos JP, Feng Jet al. Increased risk of idiopathic chronic pancreatitis in cystic fibrosis carriers. Hum Mutat 2005; 26: 303-7.

[28] Whitcomb DC. Genetic polymorphisms in alcoholic pancreatitis. Dig Dis 2005; 23: 247-54.

[29] Lindkvist B, Fajardo I, Pejler Get al. Cathepsin B activates human trypsinogen 1 but not proelastase 2 or procarboxypeptidase B. Pancreatology 2006; 6: 224-31.

[30] Apte MV, Haber PS, Norton IDet al. Alcohol and the Pancreas. Addiction Biology 1998; 3: 137-50.

[31] Sahin-Toth M, Toth M. Gain-of-function mutations associated with hereditary pancreatitis enhance autoactivation of human cationic trypsinogen. Biochem Biophys Res Commun 2000; 278: 286-9.

[32] Teich N, Rosendahl J, Toth Met al. Mutations of human cationic trypsinogen (PRSS1) and chronic pancreatitis. Hum Mutat 2006; 27: 721-30.

[33] Apte MV, Wilson JS, McCaughan GWet al. Ethanol-induced alterations in messenger RNA levels correlate with glandular content of pancreatic enzymes. Journal of Laboratory \& Clinical Medicine 1995; 125: 634-40.

[34] Ponnappa BC, Hoek JB, Waring AJet al. Effect of ethanol on amylase secretion and cellular calcium homeostasis in pancreatic acini from normal and ethanol-fed rats. Biochemical Pharmacology 1987; 36: 69-79.

[35] Siegmund E, Luthen F, Kunert Jet al. Ethanol modifies the actin cytoskeleton in rat pancreatic acinar cells--comparison with effects of CCK. Pancreatology 2004; 4: 1221.

[36] Wilson JS, Apte MV, Thomas MCet al. Effects of ethanol, acetaldehyde and cholesteryl esters on pancreatic lysosomes. Gut 1992; 33: 1099-104.

[37] Haber PS, Wilson JS, Apte MVet al. Fatty acid ethyl esters increase rat pancreatic lysosomal fragility. Journal of Laboratory \& Clinical Medicine 1993; 121: 759-64.

[38] Lange LG. Nonoxidative ethanol metabolism: formation of fatty acid ethyl esters by cholesterol esterase. Proceedings of the National Academy of Sciences of the United States of America 1982; 79: 3954-7.

[39] Wilson JS, Colley PW, Sosula Let al. Alcohol causes a fatty pancreas. A rat model of ethanol-induced pancreatic steatosis. Alcohol Clinical and Experimental Research 1982; 6: 117-21.

[40] Gukovskaya AS, Mouria M, Gukovsky Iet al. Ethanol metabolism and transcription factor activation in pancreatic acinar cells in rats. Gastroenterology 2002; 122: 10618.

[41] Haber PS, Apte MV, L. ATet al. Metabolism of ethanol by rat pancreatic acinar cells. Journal of Laboratory and Clinical Medicine 1998; 132: 294-302.

[42] Haber PS, Apte MV, Moran Cet al. Non-oxidative metabolism of ethanol by rat pancreatic acini. Pancreatology 2004; 4: 82-9.

[43] Chiang $\mathrm{CP}, \mathrm{Wu} \mathrm{CW}$, Lee SPet al. Expression pattern, ethanol-metabolizing activities, and cellular localization of alcohol and aldehyde dehydrogenases in human 
pancreas: implications for pathogenesis of alcohol-induced pancreatic injury. Alcohol Clin Exp Res 2009; 33: 1059-68.

[44] Norton I, Apte M, Haber Pet al. P4502E1 is present in rat pancreas and is induced by chronic ethanol administration. Gastroenterology 1996; 110: A1280.

[45] Ekstrom G, Ingelman-Sundberg M. Rat liver microsomal NADPH-supported oxidase activity and lipid peroxidation dependent on ethanol-inducible cytochrome P-450 (P450IIE1). Biochemical Pharmacology 1989; 38: 1313-9.

[46] Shaw S, Jayatilleke E. The role of cellular oxidases and catalytic iron in the pathogenesis of ethanol-induced liver injury. Life Sciences 1992; 50: 2045-52.

[47] Altomare E, Grattagliano I, Vendemiale Get al. Acute ethanol administration induces oxidative changes in rat pancreatic tissue. Gut 1996; 38: 742-46.

[48] Casini A, Galli A, Pignalosa Pet al. Collagen type I synthesized by pancreatic periacinar stellate cells (PSC) co-localizes with lipid peroxidation-derived aldehydes in chronic alcoholic pancreatitis. Journal of Pathology 2000; 192: 81-9.

[49] Norton ID, Apte MV, Lux Oet al. Chronic ethanol administration causes oxidative stress in the rat pancreas. Journal of Laboratory \& Clinical Medicine 1998; 131: 4426.

[50] Werner J, Saghir M, Fernandez-del Castillo Cet al. Linkage of oxidative and nonoxidative ethanol metabolism in the pancreas and toxicity of nonoxidative ethanol metabolites for pancreatic acinar cells. Surgery 2001; 129: 736-44.

[51] Werner J, Saghir M, Warshaw ALet al. Alcoholic pancreatitis in rats: injury from nonoxidative metabolites of ethanol. American Journal of Physiology Gastrointestinal and Liver Physiology 2002; 283: G65-73.

[52] Nordback IH, MacGowan S, Potter Jet al. The role of acetaldehyde in the pathogenesis of acute alcoholic pancreatitis. Annals of Surgery 1991; 214: 671-8.

[53] Sankaran H, Lewin MB, Wong Aet al. Irreversible inhibition by acetaldehyde of cholecystokinin-induced amylase secretion from isolated rat pancreatic acini. Biochem Pharmacol 1985; 34: 2859-63.

[54] Lieber CS. Metabolism of ethanol. In: Lieber CS, ed. Medical and nutritional complications of alcoholism: mechanisms and management. New York: Plenum Publishing Corporation 1992:pp 1-35.

[55] Dryden GW, Deacuic I, Arteel Get al. Clinical implications of oxidative stress and antioxidant therapy. Current Gastroenterology Reviews 2005; 7: 308-16.

[56] Lugea A, Gukovsky I, Gukovskaya ASet al. Nonoxidative ethanol metabolites alter extracellular matrix protein content in rat pancreas. Gastroenterology 2003; 125 : 1845-59.

[57] Werner J, Laposata M, Fernandez-del Castillo Cet al. Pancreatic injury in rats induced by fatty acid ethyl ester, a nonoxidative metabolite of alcohol. Gastroenterology 1997; 113: 286-94.

[58] Hungund BL, Goldstein DB, Villegas Fet al. Formation of fatty acid ethyl esters during chronic ethanol treatment in mice. Biochemical Pharmacology 1988; 37: 3001-4.

[59] Lange LG, Sobel BE. Mitochondrial dysfunction induced by fatty acid ethyl esters, myocardial metabolites of ethanol. Journal of Clinical Investigation 1983; 72: 72431.

[60] Criddle DN, Murphy J, Fistetto Get al. Fatty acid ethyl esters cause pancreatic calcium toxicity via inositol trisphosphate receptors and loss of ATP synthesis. Gastroenterology 2006; 130: 781-93.

[61] Del Castillo-Vaquero A, Salido GM, Gonzalez A. Increased calcium influx in the presence of ethanol in mouse pancreatic acinar cells. Int J Exp Pathol; 91: 114-24. 
[62] Apte M, Pirola R, Wilson J. New insights into alcoholic pancreatitis and pancreatic cancer. J Gastroenterol Hepatol 2009; 24 Suppl 3: S51-6.

[63] Apte MV, Haber PS, Applegate TLet al. Periacinar Stellate Shaped Cells in Rat Pancreas - Identification, Isolation, and Culture. Gut 1998; 43: 128-33.

[64] Bachem MG, Schneider E, Gross Het al. Identification, Culture, and Characterization of Pancreatic Stellate Cells in Rats and Humans. Gastroenterology 1998; 115: 42132.

[65] Apte MV, Haber PS, Darby SJet al. Pancreatic stellate cells are activated by proinflammatory cytokines : implications for pancreatic fibrogenesis. Gut 1999; 44: 534-41.

[66] Mews P, Phillips P, Fahmy Ret al. Pancreatic stellate cells respond to inflammatory cytokines: potential role in chronic pancreatitis. Gut 2002; 50: 535-41.

[67] Schneider E, Schmid-Kotsas A, Zhao Jet al. Identification of mediators stimulating proliferation and matrix synthesis of rat pancreatic stellate cells. American Journal of Physiology: Cell Physiology 2001; 281: C532-43.

[68] Phillips PA, McCarroll JA, Park Set al. Rat pancreatic stellate cells secrete matrix metalloproteinases: implications for extracellular matrix turnover. Gut 2003; 52: 275-82.

[69] Apte MV, Phillips PA, Fahmy RGet al. Does alcohol directly stimulate pancreatic fibrogenesis? Studies with rat pancreatic stellate cells. Gastroenterology 2000; 118 : 780-94.

[70] Masamune A, Satoh A, Watanabe Tet al. Effects of ethanol and its metabolites on human pancreatic stellate cells. Dig Dis Sci 2010; 55: 204-11.

[71] Lawrencia C, Charrier A, Huang Get al. Ethanol-mediated expression of connective tissue growth factor (CCN2) in mouse pancreatic stellate cells. Growth Factors 2009; 27: 91-9.

[72] Vonlaufen A, Phillips P, Xu Zet al. Withdrawal of alcohol promotes regression while continued alcohol intake promotes persistence of LPS-induced pancreatic injury in alcohol-fed rats. Gut 2010; in press, accepted June 2010.

[73] Vonlaufen A, Xu Z, Daniel Bet al. Bacterial endotoxin: a trigger factor for alcoholic pancreatitis? Evidence from a novel, physiologically relevant animal model. Gastroenterology 2007; 133: 1293-303.

[74] Apte MV, Pirola RC, Wilson JS. Individual susceptibility to alcoholic pancreatitis. J Gastroenterol Hepatol 2008; 23 Suppl 1: S63-8.

[75] Witt H, Apte MV, Keim Vet al. Chronic pancreatitis: challenges and advances in pathogenesis, genetics, diagnosis, and therapy. Gastroenterology 2007; 132: 1557-73.

[76] Apte MV, Pirola RC, Wilson JS. Where there's smoke there's not necessarily fire. Gut 2005; 54: 446-7.

[77] Law R, Parsi M, Lopez Ret al. Cigarette smoking is independently associated with chronic pancreatitis. Pancreatology 2010; 10: 54-9.

[78] Maisonneuve P, Lowenfels AB, Mullhaupt Bet al. Cigarette smoking accelerates progression of alcoholic chronic pancreatitis. Gut 2005; 54: 510-4.

[79] Ammann RW, Raimondi S, Maisonneuve Pet al. Is obesity an additional risk factor for alcoholic chronic pancreatitis? Pancreatology 2010; 10: 47-53.

[80] Wannamethee SG, Shaper AG. Alcohol, body weight, and weight gain in middleaged men. Am J Clin Nutr 2003; 77: 1312-7.

[81] Miyasaka K, Ohta M, Takano Set al. Carboxylester lipase gene polymorphism as a risk of alcohol-induced pancreatitis. Pancreas 2005; 30: e87-e91.

[82] Witt H, Sahin-Toth M, Landt Oet al. A degradation-sensitive anionic trypsinogen (PRSS2) variant protects against chronic pancreatitis. Nat Genet 2006; 38: 668-73. 
[83] Witt H, Luck W, Becker Met al. Mutation in the SPINK1 trypsin inhibitor gene, alcohol use, and chronic pancreatitis. Jama 2001; 285: 2716-7.

[84] Diaconu BL, Ciobanu L, Mocan Tet al. Investigation of the SPINK1 N34S mutation in Romanian patients with alcoholic chronic pancreatitis. A clinical analysis based on the criteria of the M-ANNHEIM classification. J Gastrointestin Liver Dis 2009; 18: $143-50$.

[85] Kuwata K, Hirota M, Shimizu Het al. Functional analysis of recombinant pancreatic secretory trypsin inhibitor protein with amino-acid substitution. J Gastroenterol 2002; 37: 928-34.

[86] Pandol SJ, Periskic S, Gukovsky Iet al. Ethanol diet increases the sensitivity of rats to pancreatitis induced by cholecystokinin octapeptide. Gastroenterology 1999; 117: 706-16.

[87] Bode C, Kugler V, Bode JC. Endotoxemia in patients with alcoholic and nonalcoholic cirrhosis and in subjects with no evidence of chronic liver disease following acute alcohol excess. J Hepatol 1987; 4: 8-14.

[88] Bode JC, Parlesak A, Bode C. Gut derived bacterial toxins (endotoxin) and alcohol liver disease. In: Agarwal DP, Seitz HK, eds. Alcohol in Health and Disease. New York/Basel: Marcel Dekker 2001:369-86.

[89] Ammori BJ, Leeder PC, King RFet al. Early increase in intestinal permeability in patients with severe acute pancreatitis: correlation with endotoxemia, organ failure, and mortality. J Gastrointest Surg 1999; 3: 252-62.

[90] Gullo L, Barbara L, Labo G. Effect of cessation of alcohol use on the course of pancreatic dysfunction in alcoholic pancreatitis. Gastroenterology 1988; 95: 1063-8.

[91] Wilson JS, Bernstein L, McDonald Cet al. Diet and drinking habits in relation to the development of alcoholic pancreatitis. Gut 1985; 26: 882-7.

[92] Nakamura Y, Ishikawa A, Sekiguchi Set al. Spirits increase the risk for chronic pancreatitis more than beer and Sake in 111 Japanese alcoholics. Gastroenterology 2002; 122: A509.

[93] Lowenfels AB, Zwemer FL, Jhangiani Set al. Pancreatitis in a native American Indian population. Pancreas 1987; 2: 694-7.

[94] Haber PS, Wilson JS, Pirola RC. Smoking and alcoholic pancreatitis. Pancreas 1993; 8: 568-72.

[95] Wilson JS, Gossat D, Tait Aet al. Evidence for an inherited predisposition to alcoholic pancreatitis. A controlled HLA typing study. Digestive Diseases and Sciences 1984; 29: 727-30.

[96] Haber PS, Wilson JS, McGarity BHet al. Alpha 1 antitrypsin phenotypes and alcoholic pancreatitis. Gut 1991; 32: 945-8.

[97] Norton ID, Apte MV, Dixson Het al. Cystic fibrosis genotypes and alcoholic pancreatitis. J Gastroenterol Hepatol 1998; 13: 496-500.

[98] Frenzer A, Butler WJ, Norton IDet al. Polymorphism in alcohol-metabolizing enzymes, glutathione S-transferases and apolipoprotein $\mathrm{E}$ and susceptibility to alcohol-induced cirrhosis and chronic pancreatitis. Journal of Gastroenterology and Hepatology 2002; 17: 177-82.

[99] Schneider A, Barmada MM, Slivka Aet al. Transforming growth factor-beta1, interleukin-10 and interferon-gamma cytokine polymorphisms in patients with hereditary, familial and sporadic chronic pancreatitis. Pancreatology 2004; 4: 490-4.

[100] Schneider A, Pfutzer RH, Barmada MMet al. SPINK1/PSTI gene mutations in patients with alcoholic chronic pancreatitis from North America. Gastroenterology 2002; 122: A563. 
[101] Ockenga J, Vogel A, Teich Net al. UDP glucuronosyltransferase (UGT1A7) gene polymorphisms increase the risk of chronic pancreatitis and pancreatic cancer. Gastroenterology 2003; 124: 1802-8. 


\section{Figure Legends}

Figure 1: Alcoholic chronic pancreatitis. H\&E section of the pancreas from a patient with alcohol-induced chronic pancreatitis depicting abundant fibrosis surrounding atrophic acini (Original magnification $\mathrm{x} 40$ ).

Figure 2: Necrosis-fibrosis concept of progression of acute to chronic pancreatitis. Repeated attacks of acute pancreatic necroinflammation cause increasing residual damage to the pancreas, eventually resulting in irreversible injury characterized by acinar atrophy and fibrosis.

Figure 3: Vitamin A containing lipid droplets in pancreatic stellate cells (PSCs). Phase contrast micrograph of PSCs in early culture displaying a typical polygonal shape and abundant lipid droplets in the cytoplasm surrounding the nucleus. When exposed to UV light at $380 \mathrm{~nm}$, these droplets exhibit a transient blue green fluorescence, characteristic of vitamin A. [Reprinted with permission from BMJ Group: Apte MV, Haber PS, Applegate TN, Norton ID, McCaughan GW, Korsten MA, Pirola RC, Wilson JS. Periacinar stellate shaped cells in rat pancreas: identification, isolation and culture of stellate cells from rat pancreas. Gut 1998;43:128-133].

Figure 4: Desmin positive PSCs in rat pancreas - the left panel depicts a frozen section of the pancreas immunostained for desmin, while the right panel shows a corresponding line diagram. An acinus (A) made up of individual acinar cells is surrounded by brown stained, desmin-positive PSCs with a central cell body and long cytoplasmic projections extending along the basolateral aspect of acinar cells. [Reprinted with permission from BMJ Group: Apte MV, Haber PS, Applegate TN, Norton ID, McCaughan GW, Korsten MA, Pirola RC, Wilson JS. Periacinar 
stellate shaped cells in rat pancreas: identification, isolation and culture of stellate cells from rat pancreas. Gut 1998; 43:128-133].

Figure 5: Pancreatic necroinflammation, acinar atrophy and fibrosis in a rat model of alcoholic pancreatitis. (A) Effect of alcohol \pm LPS challenge on pancreatic histology. H\&E stained pancreatic sections were assessed morphometrically for the presence and extent of acinar cell vacuolisation, acinar cell necrosis, hemorrhage, inflammatory infiltrate and edema. The histology score of alcohol-fed LPS challenged rats (AL) was significantly higher than in the other 3 groups [rats fed a control diet receiving saline injections (C); rats fed an alcohol diet receiving saline injections (A) and rats fed a control diet receiving LPS injections (CL)] (*p<0.001 AL vs $\mathrm{C} ; \# \mathrm{p}<0.01$ AL vs A; $\$ \mathrm{p}<0.05$ AL vs CL; $\mathrm{n}=8$ animals/group).

(B) Effect of alcohol \pm LPS challenge on extracellular matrix deposition in the pancreas. The Figure depicts representative Masson's trichrome stained pancreatic sections from i) rats fed a control diet (CL), and ii) rats fed an alcohol diet (AL) challenged with LPS. Obvious blue stained fibrotic bands were observed in periacinar location in the pancreas of AL rats. In addition, the glands exhibited shrunken acini. In CL animals, "blue" staining was confined to periductular and perivascular regions, and notably, acinar size was normal (Original magnification x1000).

[Figs $5 \mathrm{~A}$ and $\mathrm{B}$ reprinted with permission from Elsevier: Vonlaufen A, Xu Z, Daniel B, Kumar RK, Pirola R, Wilson J, Apte MV. Bacterial endotoxin - a trigger factor for alcoholic pancreatitis? Evidence from a novel, physiologically relevant animal model Gastroenterology 2007;133: 1293-301]

Figure 6: Reversal of collagen deposition after established pancreatitis. The graph depicts results of morphometric analysis of collagen staining (using Sirius Red) in pancreatic sections from four 
groups of animals : a) CL - control diet fed rats challenged with LPS; b) AL alcohol-fed rats challenged with LPS; c) AL+A alcohol-diet fed rats challenged with LPS, then continued on alcohol for 3 weeks; d) AL+C alcohol-diet fed rats challenged with LPS, with withdrawal of alcohol for 3 weeks. AL animals had significantly increased collagen staining compared to CL animals. Continuation of alcohol feeding after established pancreatitis (AL+A) resulted in a persistent increase in collagen staining. Withdrawal of alcohol after established pancreatitis $(\mathrm{AL}+\mathrm{C})$ resulted in a significant decrease in collagen staining to control (CL) levels. $\mathrm{p}<0.01 \mathrm{AL}$ and $\mathrm{AL}+\mathrm{A}$ vs $\mathrm{CL}$ and $\mathrm{AL}+\mathrm{C} ; \mathrm{n}=10$ rats / group.

Figure 7: Current concept of the pathogenesis of alcoholic pancreatitis. The Figure depicts An acinar cell that is affected by alcohol and its metabolites such that it is susceptible to autodigestive injury, necroinflammation and cell death via :

1) increased digestive and lysosomal enzyme content secondary to increased synthesis (increased mRNA) and impaired secretion, and

2) destabilization of lysosomes and zymogen granules, a process mediated by oxidant stress, ROS, cholesteryl esters (CE) and FAEE and decreased GP2

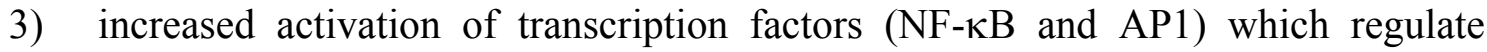
cytokine expression

4) a sustained increase in cytoplasmic calcium and mitochondrial calcium overload leading to mitochondrial depolarisation

In the presence of an appropriate trigger factor such as bacterial endotoxin (LPS), overt necroinflammation (acute pancreatitis) is initiated. For abbreviations, see text.

A pancreatic stellate cell that is activated by :

a) direct effects of alcohol, its metabolites and oxidant stress,

b) endotoxin (LPS) 
b) exogenous cytokines released during pancreatic necroinflammation or endogenous cytokines released in response to alcohol exposure

leading to excessive extracellular matrix protein production and pancreatic fibrosis (chronic pancreatitis).

Abbreviations : Ac - acetaldehyde; CE - cholesteryl esters; FAEE - fatty acid ethyl esters; L - lysosome; mRNA - messenger RNA; ROS - reactive oxygen species; ZG - zymogen granule. 
Table

Individual Susceptibility to Alcoholic Pancreatitis

\begin{tabular}{|c|c|c|}
\hline Factor & & Association \\
\hline Drinking pattern & No & Wilson et al, $1985^{91}$ \\
\hline \multirow[t]{2}{*}{ Beverage type } & & Wilson et al, $1985^{91}$ \\
\hline & Yes & *Nakamura et al, $2002^{92}$ \\
\hline Diet & No & Wilson et al, $1985^{91}$ \\
\hline \multirow[t]{2}{*}{ Smoking } & Yes & Lowenfels et al, $1987^{93}$ \\
\hline & No & Haber et al, $1993^{94}$ \\
\hline Obesity & Yes & *Ammann et al, $2010^{79}$ \\
\hline \multicolumn{3}{|l|}{ Inherited factors } \\
\hline HLA & No & Wilson et al, $1984{ }^{95}$ \\
\hline$\alpha 1$-antitrypsin deficiency & No & Haber et al, $1993^{96}$ \\
\hline Cystic fibrosis genotype & No & Norton et al, $1994^{97}$ \\
\hline Cytochrome P4502E1 polymorphism & No & Frenzer et al, $2002^{98}$ \\
\hline ADH genotype & No & Frenzer et al, $2002^{98}$ \\
\hline Anionic trypsinogen gene mutation & Yes & *Witt et al, $2006^{82}$ \\
\hline PSTI/SPINK1 mutations & Yes & Witt et al, $2001^{83}$ \\
\hline TNF $\alpha$, TGF $\beta$, IL10 polymorphisms & No & ${ }^{*}$ Schneider et al, $2004{ }^{99}$ \\
\hline $\begin{array}{l}\text { Detoxifying enzymes } \\
\quad \text { - Glutathione S-transferase }\end{array}$ & No & $\begin{array}{l}\text { Frenzer et al, } 2002^{98} \\
*^{*} \text { Schneider et al, } 2002^{100}\end{array}$ \\
\hline - UDP-glucuronosyl transferase & Yes & ${ }^{*}$ Ockenga et al, $2003{ }^{101}$ \\
\hline Cholestery ester lipase polymorphism & Yes & Miyasaka et al, $2005^{81}$ \\
\hline
\end{tabular}

* Studies which did not include alcoholics without pancreatitis as controls 